\title{
Lorentz violations and Euclidean signature metrics
}

\author{
J. Fernando Barbero G.* and Eduardo J. S. Villaseñor ${ }^{\dagger}$ \\ Instituto de Matemáticas y Física Fundamental, Centro de Física Miguel A. Catalán, CSIC, Serrano 113bis, 28006 Madrid, Spain
}

(Received 24 July 2003; published 30 October 2003)

\begin{abstract}
We show that the families of effective actions considered by Jacobson et al. to study Lorentz invariance violations contain a class of models that represent pure general relativity with a Euclidean signature. We also point out that some members of this family of actions preserve Lorentz invariance in a generalized sense.
\end{abstract}

DOI: 10.1103/PhysRevD.68.087501

PACS number(s): 04.20.Cv

In recent years there have been several proposals to study Lorentz invariance violations in general relativity and their observational consequences (see [1-3] and references therein). The main ingredient of these models is the introduction of a preferred frame (referred to by the authors as the aether) described by a unit timelike vector field $u^{a}$. In order to preserve general covariance $u^{a}$ is taken as a dynamical field. The most general action considered in these papers has the form

$$
\begin{aligned}
\mathcal{L}_{g, u}= & a_{0}-a_{1} R-a_{2} R_{a b} u^{a} u^{b}-b_{1} F^{a b} F_{a b}-b_{2}\left(\nabla_{a} u_{b}\right)\left(\nabla^{a} u^{b}\right) \\
& -b_{3} \dot{u}^{a} \dot{u}_{a}+\lambda\left(g^{a b} u_{a} u_{b}-1\right),
\end{aligned}
$$

where $\dot{u}^{a}:=u^{m} \nabla_{m} u^{a}, \lambda$ is the Lagrange multiplier that enforces the condition that $u^{a}$ is a unit vector, and $F_{a b}$ is defined as $F_{a b}:=2 \nabla_{[a} u_{b]}$. It is important to notice that the models described by Eq. (1) are not the usual tensor-vector theories due to this constraint. This type of Lagrangian has already been considered in the literature by Kostelecký and Samuel [4] for gravitational models and by Kostelecký and Mewes [5] in the context of electrodynamics. The role of questions similar to the ones discussed here, in particular, coordinate invariance, in the construction of dispersion relations with physical Lorentz violation is discussed in [6].

We want to point out here that some of these actions can be interpreted as describing pure general relativity with a Euclidean signature and others are, in fact, equivalent to Lorentzian general relativity without any Lorentz violating effects.

Following the ideas presented in [7], let us consider the metric

$$
g_{a b}^{E}=-\frac{1}{2} \sqrt{|\alpha(\alpha+2 \beta)|}\left[g_{a b}-2 \frac{\alpha+\beta}{\alpha+2 \beta} u_{a} u_{b}\right],
$$

where $\alpha$ and $\beta$ are two real parameters, $g_{a b}$ is a Lorentzian metric [with $(+---)$ signature], and $u^{a}$ is a unit timelike vector field $\left(g_{a b} u^{a} u^{b}=1\right)$. Here $u_{a} \equiv g_{a b} u^{a}$. If we compute the determinant of $g_{a b}^{E}$ we obtain

$$
g^{E} \equiv \operatorname{det}_{a b}^{E}=-\frac{1}{16} \alpha^{3}(\alpha+2 \beta) \operatorname{det}_{a b} .
$$

\footnotetext{
*Electronic address: jfbarbero@imaff.cfmac.csic.es

†Electronic address: eduardo@imaff.cfmac.csic.es
}

As we can see the fact that $u^{a}$ is a unit vector implies that the determinants of $g_{a b}^{E}$ and $g_{a b}$ are proportional to each other with a constant of proportionality that can be made either positive or negative by choosing appropriate values of $\alpha$ and $\beta$. Let us write now the Einstein-Hilbert action for $g_{a b}^{E}$ as a function of $g_{a b}$ and $u^{a}$. To this end we need the inverse metric $g^{E a b}$ and the Christoffel symbols for $g_{a b}^{E}$ (here $g^{a b}$ satisfies $g_{a b} g^{b c}=\delta_{a}^{c}$ ):

$$
\begin{aligned}
g^{E a b}= & -\frac{2}{\sqrt{|\alpha(\alpha+2 \beta)|}}\left[g^{a b}-2 \frac{\alpha+\beta}{\alpha} u^{a} u^{b}\right] \\
\Gamma_{b c}^{E a}= & \Gamma_{b c}^{a}-\frac{\alpha+\beta}{\alpha+2 \beta}\left[\nabla_{b}\left(u^{a} u_{c}\right)+\nabla_{c}\left(u^{a} u_{b}\right)-\nabla^{a}\left(u_{b} u_{c}\right)\right] \\
& +\frac{2(\alpha+\beta)^{2}}{\alpha(\alpha+2 \beta)}\left[u^{a} \nabla_{b} u_{c}+u^{a} \nabla_{c} u_{b}-u^{a} u^{d} \nabla_{d}\left(u_{b} u_{c}\right)\right] .
\end{aligned}
$$

A tedious but straightforward computation now gives

$$
\begin{aligned}
S_{E}= & \int d^{4} x \sqrt{\left|g_{E}\right|} g^{E a b} R_{a b}^{E} \\
= & \operatorname{sgn}(\alpha) \int d^{4} x \sqrt{|g|}\left[-\frac{\alpha}{2} R+(\alpha+\beta) u^{a} u^{b} R_{a b}\right. \\
& \left.-\frac{(\alpha+\beta)^{2}}{\alpha+2 \beta} g^{a b} \omega_{a} \omega_{b}\right],
\end{aligned}
$$

where $R_{a b}^{E}$ is the Ricci tensor ${ }^{1}$ built with $g_{a b}^{E}, R_{a b}$, and $R$ with $g_{a b}$, and $\omega_{a}$ is the twist of $u^{a}$ given by

$$
\omega_{a}=\epsilon_{a a_{1} a_{2} a_{3}} u^{a_{1}} \nabla^{a_{2}} u^{a_{3}} .
$$

It is useful to notice that

$$
\begin{aligned}
\omega_{a} \omega^{a} & =\left(\nabla_{a} u_{b}\right)\left(\nabla^{a} u^{b}\right)-\left(\nabla_{a} u_{b}\right)\left(\nabla^{b} u^{a}\right)-\dot{u}^{a} \dot{u}_{a} \\
& =\frac{1}{2} F_{a b} F^{a b}-\dot{u}^{a} \dot{u}_{a} .
\end{aligned}
$$

\footnotetext{
${ }^{1}$ Throughout this paper we are using the conventions of Wald [8] for the definitions of geometric objects and, in particular, for the Riemann tensor.
} 
As in Eq. (1), the condition that $u^{a}$ is a unit vector can be explicitly incorporated into the action by adding a suitable Lagrange multiplier term to Eq. (6). Another way to do that [7] is to write $u^{a}=\eta^{a} /\left(g_{b c} \eta^{b} \eta^{c}\right)^{1 / 2}$, with an unconstrained, timelike, vector field $\eta^{a}$; in which case the action becomes invariant under the gauge transformations consisting in local rescalings of the vector field. We can readily see that Eq. (6) is a particular case of the action (1) considered in [1] with the parameter choices $a_{0}=0, \quad a_{1}=|\alpha / 2|, \quad a_{2}=-\operatorname{sgn}(\alpha)(\alpha$ $+\beta), \quad b_{1}=(\operatorname{sgn} \alpha)(\alpha+\beta)^{2} / 2(\alpha+2 \beta), \quad b_{2}=0, \quad$ and $\quad b_{3}$ $=-2 b_{1}$.

Several comments are now in order.

(i) Some of the parameter choices do not change the signature of the metric. If both $g_{a b}^{E}$ and $g_{a b}$ have Lorentzian signatures, the action (6) is strictly equivalent to the Einstein-Hilbert action for $g_{a b}$. It is important to realize that Eq. (6) has a gauge symmetry that is related to the fact that the variations in the vector field can always be compensated inside $g_{a b}^{E}$ by a suitable variation of the metric $g_{a b}$. This also means that the field equations coming from variations in the vector field are always redundant. We see then that there is a one to one correspondence between the solutions to the field equations for the Einstein-Hilbert action (Lorentzian or Euclidean) and gauge equivalence classes of solutions to the field equations derived from Eq. (6). In our opinion, it would not be justified to talk about Lorentz violating effects when $g_{a b}^{E}$ is Lorentzian.

(ii) The fact that $u^{a}$ is dynamical or not is irrelevant in our scheme. If $u^{a}$ is a fixed geometric structure, general covariance is broken but, as long as matter couples to $g_{a b}^{E}$, the physical content of the model corresponds to general relativity in the sense that there is a one to one correspondence between the solutions of the two theories and their symmetries.

(iii) Because of the presence of two different metrics $g_{a b}^{E}$ and $g_{a b}$, one can consider matter couplings to either of them. If matter is coupled to $g_{a b}^{E}$ and the parameters of the model are chosen in such a way that $g_{a b}^{E}$ is Lorentzian, we still have Lorentzian general relativity without breaking any Lorentz invariance in the sense discussed above. If, on the other hand, we choose the parameters to get a Euclidean signature we end up with Euclidean general relativity with matter. Finally, if matter is coupled to $g_{a b}$ we have the Lorentz violating effects described in [1-3].

(iv) The field equations obtained by varying in $u^{a}$ are redundant. This can be explicitly checked by varying our action (6) with respect to $u^{a}$ and checking that the equations thus obtained are satisfied as a consequence of the equations derived by varying with respect to $g_{a b}$. This can also be seen by noticing that a variation in $g_{a b}^{E}$ of the type generated by changing $u^{a}$ can also be obtained by a suitable variation of $g_{a b}$, as discussed above.

(v) If $u^{a}$ is hypersurface orthogonal the twist is not present, and we get the formulation presented in [7] in the context of real Wick rotations.

The authors wish to thank G. Mena and T. Jacobson for valuable comments. This work was supported by the Spanish MCYT under the research project BFM2002-04031-C02-02. E.J.S.V. is supported by the Spanish Ministry of Education and Culture and cofinanced by the European Social Fund.
[1] T. Jacobson and D. Mattingly, Phys. Rev. D 64, 024028 (2001).

[2] T. Jacobson, S. Liberati, and D. Mattingly, gr-qc/0303001.

[3] D. Mattingly and T. Jacobson, gr-qc/0112012.

[4] V.A. Kostelecký and S. Samuel, Phys. Rev. D 40, 1886 (1989).
[5] V.A. Kostelecký and M. Mewes, Phys. Rev. D 66, 056005 (2002).

[6] R. Lehnert, Phys. Rev. D 68, 085003 (2003).

[7] J.F. Barbero G., Phys. Rev. D 54, 1492 (1996).

[8] R. M. Wald, General Relativity (University of Chicago Press, Chicago, 1984). 